\title{
Gradhiva
}

GRADHIV

Revue d'anthropologie et d'histoire des arts

28 | 2018

Capsules temporelles

\section{Des seins de moine à Vézelay. Eugène-Eugénie, nouvelle image transgenre au XII ${ }^{\mathrm{e}}$ siècle}

A monk's breasts in Vézelay. Eugene-Eugenia, a new transgender picture in the XII ${ }^{\text {th }}$ century

\section{Chloé Maillet}

\section{(2) OpenEdition}

\section{Journals}

Édition électronique

URL : http://journals.openedition.org/gradhiva/3897

DOI : 10.4000/gradhiva.3897

ISSN : 1760-849X

Éditeur

Musée du quai Branly Jacques Chirac

Édition imprimée

Date de publication : 28 novembre 2018

Pagination : 220-243

ISBN : 978-2-35744-111-8

ISSN : 0764-8928

Référence électronique

Chloé Maillet, « Des seins de moine à Vézelay. Eugène-Eugénie, nouvelle image transgenre au x॥le siècle », Gradhiva [En ligne], 28 | 2018, mis en ligne le 28 novembre 2020, consulté le 07 janvier 2021. URL : http://journals.openedition.org/gradhiva/3897 ; DOI : https://doi.org/10.4000/gradhiva 3897 


\section{Des seins de moine à Vézelay}

Eugène-Eugénie, nouvelle image transgenre au xII siècle

par Chloé Maillet

Eugène-Eugénie est un·e saint·e légendaire dont le culte se développe depuis l'Antiquité tardive dans l'Empire d'Occident et d'Orient. Au x\| siècle, ce personnage figure en habit de moine mais découvrant ses seins, sur un des chapiteaux de la nef de la Madeleine de Vézelay, une église de pèlerinage très fréquentée par toute la chrétienté occidentale. Cet article vise à détricoter la construction d'une image des variations de genre en partant du succès de cette iconographie au Moyen Âge central et à interroger les limites de la binarité de genre avant l'avènement du naturalisme. 
1. La question du genre est devenue majeure dans les problèmes de l'Église depuis la fin des années 1990 et l'adoption de la loi sur le pacte civil de solidarité (Pacs) ouvert depuis 1999 aux couples quelle que soit leur orientation sexuelle. Les débats autour de la réforme du mariage en 2012 ont accentué les litiges entre scientifiques et croyants autour de ces questions alors qu'un groupe de chercheurs avait proposé un rapport historique et social sur la filiation publié en 2014 (Théry et Meroyer 2014). En 2016, sur le portail Internet de l'Église catholique de Paris une rubrique intitulée «théorie du genre " contenait plusieurs articles défendant l'idée que le genre est une construction intellectuelle sans fondement naturel et invitait les parents à se renseigner pour savoir si elle était enseignée dans l'école de leurs enfants et assortie de cours d'éducation sexuelle. En 2018, le site a amplifié sa rubrique par une chronologie de la théorie du genre et de sa légalisation (www.paris. catholique.fr/chronologiede-la-theorie-du-genre. html, consulté le 16 juillet 2018). Ironiquement,

ce débat advient alors que la notion de genre a été critiquée par les féministes pour la manière dont elle réitère la distinction nature/ culture (Butler 2005 [1990]; Haraway 1991) et que de nombreux chercheurs, dans la lignée de Donna J. Haraway et d'Isabelle Stenghers, n'envisagent plus les catégories de sexe et de genre comme une possibilité "culturelle" de contrecarrer la «nature».
2. L'absence de conformité entre apparence extérieure et être intérieur était vue très négativement (Pastoureau 2012). Il était aussi interdit à une personne assignée femme de porter les vêtements de l'autre genre, comme annoncé par le

Deutéronome:

«Une femme ne portera point un habillement d'homme, et un homme ne mettra point des vêtements de femme; car quiconque fait ces choses est en abomination à l'Éternel, ton Dieu » (Dt 22.5).

La notion de déguisement ne pouvait être acceptée que dans des contextes bien déterminés, circonscrits dans le temps et ritualisés comme le charivari, qui procède par inversion de réalités destinées à retourner à la norme une fois le rituel achevé (Le Goff et Schmitt 1981). Les rituels de travestissement ont été largement étudiés par les anthropologues à partir des analyses pionnières de Bateson (Bateson,

Severi et Houseman 1986) repensées par Carlo Severi et Michael Houseman (Houseman et Severi 1994). Dans le cas d'Eugénie-Eugène, on est bien loin d'une phase de travestissement rituel, puisqu'il s'agit bien d'un changement d'identité en conformité avec un idéal de vie monastique.

3. Suivant la catégorisation proposée par Philippe Descola, discutée par les médiévistes, le naturalisme interviendrait entre le XIV et le $x v l^{e}$ siècle, et le monde médiéval pourrait être globalement assimilé aux sociétés analogistes (Dittmar 2010). Avant cette période, l'on parle par exemple de nature féminine, mais il s'agit plus d'une catégorie générale comprenant un ensemble de phénomènes liés par analogie (faiblesse, péché, fausseté...) une certaine relation au monde (lune) soleil comme féminin/ masculin) que d'une identité de genre.
On rencontre sur un chapiteau de la nef de la basilique Sainte-MarieMadeleine de Vézelay un moine ouvrant son habit pour montrer ses seins, sous les yeux d'un juge et d'une femme en civil. II s'agit d'un personnage saint décrit comme ayant vécu une partie de sa vie sous une identité sociale féminine, puis une autre en tant qu'homme. Cette sculpture inédite prend d'autant plus d'importance qu'elle apparaît dans une des églises les plus visitées de France: Vézelay, lieu d'«émotion patrimoniale», pour reprendre les termes de Daniel Fabre (Fabre et Arnaud [dir.] 2013), est aujourd'hui une petite ville où une rue flanquée de boutiques de souvenirs, d'artisanat chrétien et de librairies religieuses accueille pèlerins et curieux. Tout est fait pour que l'expérience de la rencontre avec l'église soit celle d'une continuité ressentie entre christianisme médiéval et croyances actuelles. Pourtant le monde chrétien du $\mathrm{XI}^{\mathrm{e}}$ siècle, pas plus que son dogme ou ses images, ne ressemblent à ceux du XXI ${ }^{\mathrm{e}}$ siècle ${ }^{1}$.

L'idée qu'une personne assignée femme à la naissance puisse vivre selon une identité sociale masculine, et ce pendant toute une vie, est devenue réalité pour de nombreuses personnes au début du $\mathrm{XXI}^{\mathrm{e}}$ siècle. En revanche, qu'un tel changement d'identité ait été possible au Moyen Âge nous surprend, tant la binarité de genre semble intrinsèque au christianisme contemporain. Pourtant, depuis la fin de l'Antiquité chrétienne, les exemples, romancés et/ou réels, de personnes assignées femmes ayant volontairement ou non pris l'habit et l'identité d'hommes, parfois durant leur vie entière, sont bien identifiés. Des chercheur.se·s, souvent féministes, les ont sorti·e.s des archives où ils-elles vivaient dissimulé·e.s depuis que la critique chrétienne moderne s'était efforcée de faire passer ces cas pour des fables datées auxquelles on ne pouvait guère accorder de crédit. Marie Delcourt et Évelyne Patlagean furent les premières à les analyser sous l'angle psychanalytique et historique, suivies par les historiennes Valerie H. Hotchkiss (1996) pour l'époque médiévale et Rudolf M. Dekker, Lotte C. Van de Pol (1989) et Sylvie Steinberg (2001) pour l'époque moderne. Nommé·e.s travesti·e·s, l'historiographie leur a d'abord prêté une performativité temporaire, portant essentiellement sur un changement vestimentaire. On pourrait désormais leur appliquer le terme «transgenre», pas plus médiéval que «travestissement » (apparu $\mathrm{au} x \mathrm{xl}{ }^{\mathrm{e}}$ siècle), mais sans doute plus proche du vécu de nos contemporains, et plus conforme aux représentations du monde médiéval qui regardait toute forme de déguisement avec beaucoup de suspicion ${ }^{2}$.

Au Moyen Âge, les mots et les concepts qui désignent sexe et genre étaient différents de leurs acceptions contemporaines (Haraway 1991; Scott 2010; Butler 2005 [1990]). La distinction entre hommes et femmes est alors systématiquement liée à un problème théorique et normatif: celui de la chute et de la tentation d'Ève. D'où une vision générale de l'humanité qui nous semble excessivement misogyne, mais qu'il convient de penser dans un cadre binaire et hiérarchisé où les concepts genrés de masculin et de féminin englobent plus que la simple définition des personnes par leurs organes génitaux (Klapisch-Zuber 1999; Lett 2013). Dans cette période qu'on peut désigner comme précédant l'avènement du naturalisme ${ }^{3}$, les mots sexe (sexus) et genre (genus) n'étaient pas employés tout à fait de la même manière qu'aujourd'hui. Genus pouvait aussi bien servir à nommer les organes sexuels que le genre (masculin/féminin) et l'espèce. Sexus pouvait également prendre le sens de genre - comme dans l'expression sexus perfectior («sexe parfait») 


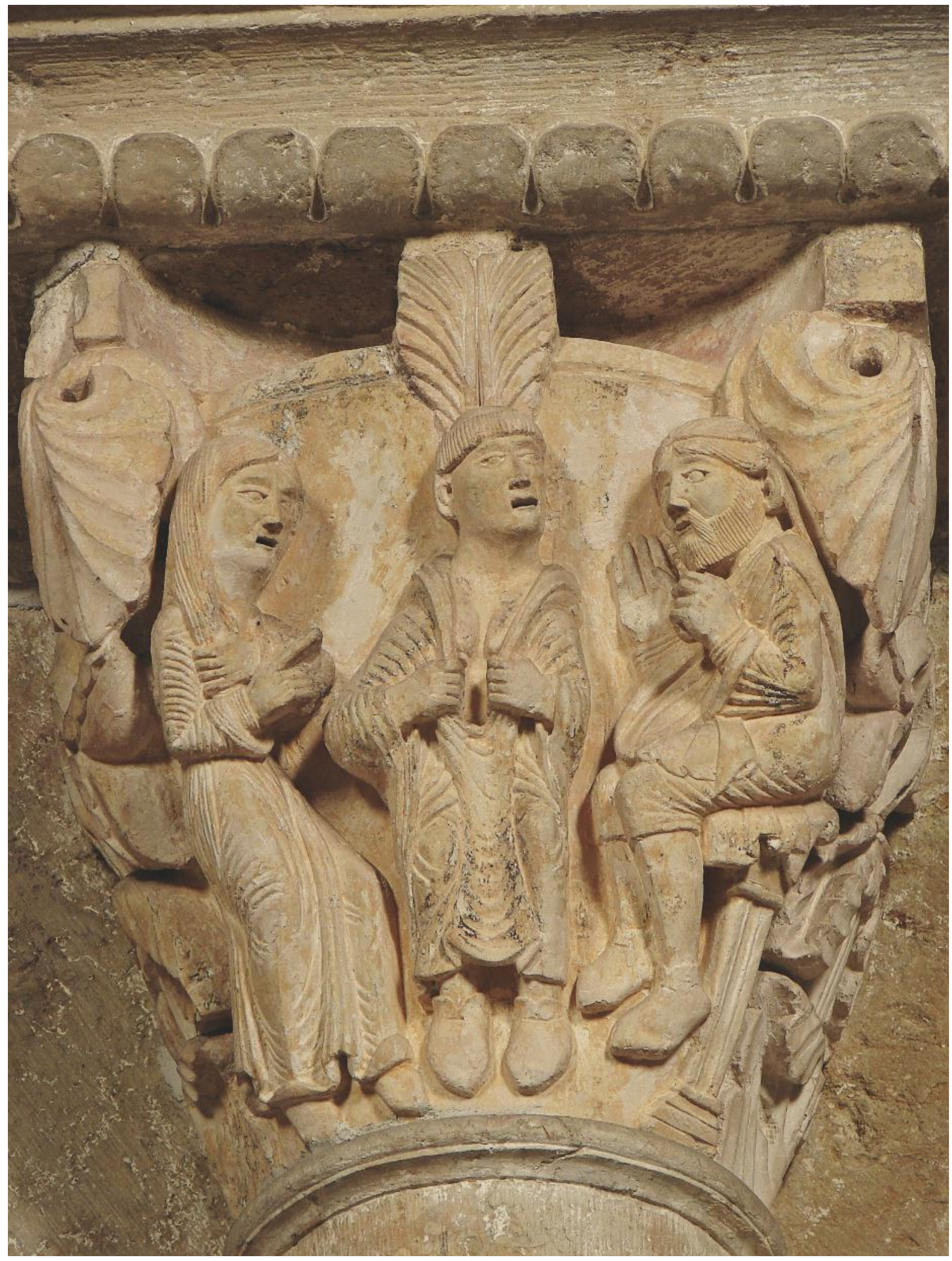


4. Ces opérations sont le fait de chirurgiens qu ont tendance à s'opposer aux médecins au cours du $\mathrm{XIV}^{\mathrm{e}}$ siècle, en promouvant une pratique intrusive. Un exemple détaillé d'opération de réassignation sexuelle peut être trouvé dans un manuscrit persan enluminé du $x v^{e}$ siècle: Haraf al-dîn, Cerrâh nâma, Paris, BNF, supplément turc 693 (Maillet 2018).

5. On voit apparaitre l'opposition entre Nature et volonté divine dans le Roman de silence, mais aussi dans Tristan de Nanteuil et Ide et Olive, continuation de la chanson de geste de la seconde moitié du XIII ${ }^{e}$ siècle, Huon de Bordeaux, comprenant la plus manifeste des expériences transgenres où la personne portant l'habit d'homme se voit miraculeusement dotée d'un pénis afin de passer une épreuve auprès de sa future fiancée et de pouvoir l'épouser (Brewka 1977; Perret 1985; Maillet 2018).

\section{page précédente}

fig. 1

Eugene-Eugénie accusée Vézelay, basilique

Sainte-Marie-Madeleine, chapiteau 59.

(C) CESCM Poitiers,

Photothèque - S. Biay. pour désigner le genre masculin - mais désignait plus couramment les organes génitaux. D'ailleurs, il n'existait pas deux sexes mais trois. Les textes de loi reprennent tous en effet la formule latine: Omnes homines aut sunt masculi aut feminae aut hermaphroditi («Tous les hommes sont ou masculins ou féminins ou hermaphrodites") (Rolker 2014). Une étude des sources normatives montre que d'un point vue moral, il n'y en avait que deux, masculin et féminin étant envisagés comme équivalents d'un système hiérarchique positif/négatif, et pas nécessairement liés à la perception sociale d'une personne comme femme ou homme. Dans le monde byzantin, existait également de manière tout à fait officielle un troisième sexe, celui des eunuques, qui comme les anges, n'étaient pas assimilables à une binarité sexuée (Sideris 2010). Il était en outre possible, dans une certaine mesure, de changer d'organes génitaux puisque des opérations pouvaient parfois être pratiquées sur des personnes intersexes, adultes et consentantes, alors appelées «hermaphrodites" (Cadden 1995; DeVun 2015) ${ }^{4}$. II n'était pas non plus impossible de changer de genre ou de rester dans une forme d'ambiguité où sexus et genus se superposaient.

II existe donc une différence entre les deux termes, mais elle est plus fine et subtile que la simple distinction entre sexe biologique et genre social, qui n'a pas de sens en dehors d'une conception moderne de nature/culture, ou «natureculture» pour reprendre l'expression de Donna Haraway (1989). La nature, au $\mathrm{xl}^{\mathrm{e}}$ siècle, était synonyme de création divine et englobait l'homme et sa culture. L'allégorie de la Nature telle qu'elle apparaît dans les romans du XIII' siècle, notamment ceux où le personnage principal, assigné femme, est devenu garçon chevalier, ne répond pas à une vision essentialiste: la Nature n'y est qu'une force parmi d'autres qui façonnent les humains, avec Noreture (l'éducation) et sous l'autorité divine ${ }^{5}$.

Dans le monde roman contemporain de Sainte-Marie-Madeleine de Vézelay, les femmes détenaient un réel pouvoir d'action et de décision politico-théologique: c'est celui que Georges Duby a mis à l'honneur dans ses Dames du XII siècle, où Aliénor d'Aquitaine côtoie Hildegarde de Bingen (Duby 1995). Le XII ${ }^{e}$ siècle est aussi une époque d'affirmation sans précédent du pouvoir de l'Église, qui impose la Réforme de l'Église, le célibat des prêtres, la division de la société entre laïcs et clercs, et exclut tous ceux qui ne rentrent pas dans le rang (prêtres mariés, clergé avide de richesses, hérétiques, juifs et musulmans) (logna-Prat 2004). Des personnes réputées "viriles», parfois appelées viragines (virago au singulier), dirigeaient des armées (Mathilde de Canossa). Plus d'une trentaine de personnes saintes ont même fait leur parcours de gloire en portant un habit masculin, l'Église les ayant reconnues et leur ayant rendu un culte (Eugénie-Eugène, Marine-Marin, Théodora-Théodore, etc.). La recherche n'est encore qu'aux prémices d'une histoire visuelle et anthropologique de la notion de transgenre au Moyen Âge; ce chapiteau de Vézelay, son sens au sein de la topologie de l'église, nous conduira aux fondements de la construction du genre au XII siècle.

\section{Montrer Eugène-Eugénie}

Lorsque l'on chemine dans la basilique Sainte-Marie-Madeleine de Vézelay, on aperçoit, dans le bas-côté nord, le chapiteau où Eugène-Eugénie ouvre son vêtement (fig. 1). 
Ainsi se présente Eugène-Eugénie (Eugenia/Eugenios/Eugenius). Par quel chemin est-il-elle arrivée jusqu'en Occident? L'histoire est encore assez méconnue. Sa $\mathrm{Vie}^{6}$ existe en grec et est associée à un culte d'Alexandrie dans l'entourage de Philippe, proconsul en Égypte sous l'empereur Commode. Très tôt traduite en latin, cette version mentionne son voyage à Rome où il-elle subit le martyre en $257^{7}$. Par ailleurs, la catacombe romaine d'Aproniano, datée du III' siècle, sur la via Latina, est réputée conserver les restes d'EugèneEugénie (Bartolini 2010). La mise par écrit de sa Vie daterait du vile ou VII siècle (Hotchkiss 1996: 134). Sa fête étant datée au 25 décembre selon le martyrologe romain, elle souffrait d'une sérieuse concurrence. C'est pourquoi elle fut très tôt associée au culte de Prothe et Hyacinthe, ses compagnons, et ainsi fêtée plus facilement le 11 septembre. Prothe et Hyacinthe, qui étaient tous deux eunuques, lui offraient déjà un modèle d'identité de genre dépassant la dualité homme/femme. Séduits par sa Vie, les encyclopédistes du XIII ${ }^{e}$ siècle l'ont insérée dans les légendiers hagiograhiques de Barthélémy de Trente, Jean de Mailly ${ }^{8}$ et Jacques de Voragine. Ce dernier fut le plus diffusé grâce à La Légende dorée qui contribua à son succès à la fin du Moyen Âge (Jacques de Voragine 2004 : chap. 130).

Le propos n'est pas ici de présenter une étude comparative de toutes les versions de la Vie. Dans l'ensemble, le récit y obéit à une même trame: 1. la conversion; 2 . le changement de genre; 3 . la fausse accusation; 4. l'exposition des seins; 5. le martyre. Fille du gouverneur Philippe, Eugénie s'était convertie au christianisme après des études poussées de philosophie avec Prothe et Hyacinthe, deux frères eunuques déjà chrétiens. Attirée par le monastère du futur saint Hélénus, réputé pour son rigorisme, la jeune personne se coupa les cheveux, prit l'habit d'homme, le nom d'Eugène et se fit accepter auprès de l'abbé Hélénus en tant que moine. Ce dernier, qui aurait été miraculeusement informé du changement de genre, aurait accepté le novice au monastère. Une femme nommée Mélancie, parfois désignée comme Mélanie, fit des avances sexuelles non équivoques à Eugène (une invitation à la rejoindre dans son lit ou une étreinte forcée, selon les versions). Face au refus, la femme accusa le frère Eugène de viol. II fut alors porté en justice devant un gouverneur qui n'était autre que son propre père, Philippe. Là, devant les yeux étonnés de la foule et de son père qui ne la reconnaissait pas, il ouvrit son habit de moine et découvrit un corps qui apparut à tous comme pourvu de seins. Blanchi.e de l'accusation (on ne pense pas qu'une femme puisse en violer une autre), le moine redevenu Eugénie convertit ses parents par ce geste; ils subirent ensemble le martyre lors d'une persécution chrétienne postérieure.

Si la vie d'Eugène-Eugénie et son changement de genre social étaient bien connus et diffusés en Orient comme en Occident, toutes les images avant Vézelay la montraient ayant recouvré son identité féminine après le procès. Elle était décrite en tant que sainte Eugénie, et les images se conformaient à cette idée en la présentant en habit de femme, des habits qu'elle avait peu portés selon son histoire. Il existe d'autres images antérieures de la sainte, venant du monde byzantin ${ }^{9}$ ou du monde latin ${ }^{10}$. Les images byzantines datant d'après l'épisode iconoclaste - les seules qui furent conservées car les périodes iconoclastes (726-843) ont provoqué la destruction de la quasi-totalité des images préexistantes - la représentent invariablement en femme, sans la moindre caractéristique masculine. Même les images les plus précoces,
6. On appelle en hagiographie Vie/vita le récit de la vie d'un·e saint.e ou d'une personne que l'on a souhaité canoniser. La Vie grecque d’Eugénie est répertoriée par la Bibliotheca hagiographica greca (BHG), sous le numéro 607w-08b, et la Bibliotheca hagiographica orientalis

(BHO), sous

le numéro 281-284.

7. Bibliotheca

hagiographica latina (BHL) 2666, Vita lisible dans la Patrologie latine, sans identification d'auteur PL 21, 1105-1122, et PL 73, col 605-620

8. Jean de Mailly dans son Abbreviatio in gestis et Miraculis Sanctorum (Abbrégé des gestes et miracles des saints, 1234) place la vie d'EugèneEugénie au 25 décembre et fait un renvoi à sa vie au 11 novembre.

Jean de Mailly, dominicain de Metz, est le premier à avoir écrit des abrégés des vies de saints au XIII ${ }^{e}$ siècle et a inspiré Jacques de Voragine, Barthélémy de Trente et Vincent de Beauvais qui se sont illustrés dans ce style à grand succès (Boureau 1997 ; Maillet 2014 : chap. I).

9. Bibliothèque apostolique vaticane (BAV), Gr 1613, fol 270, BAV gr. 1156, fol 278r, Mount Athos, Mon. Laura delta 51. Une église lui est aussi dédiée à Parenzo (Croatie) : la basilique euphrasienne, comprenant des mosaïques paléochrétiennes.

10. Naples, fresque du VII ${ }^{e}$ siècle citée par Kirk Ambrose (Ambrose 2006). 


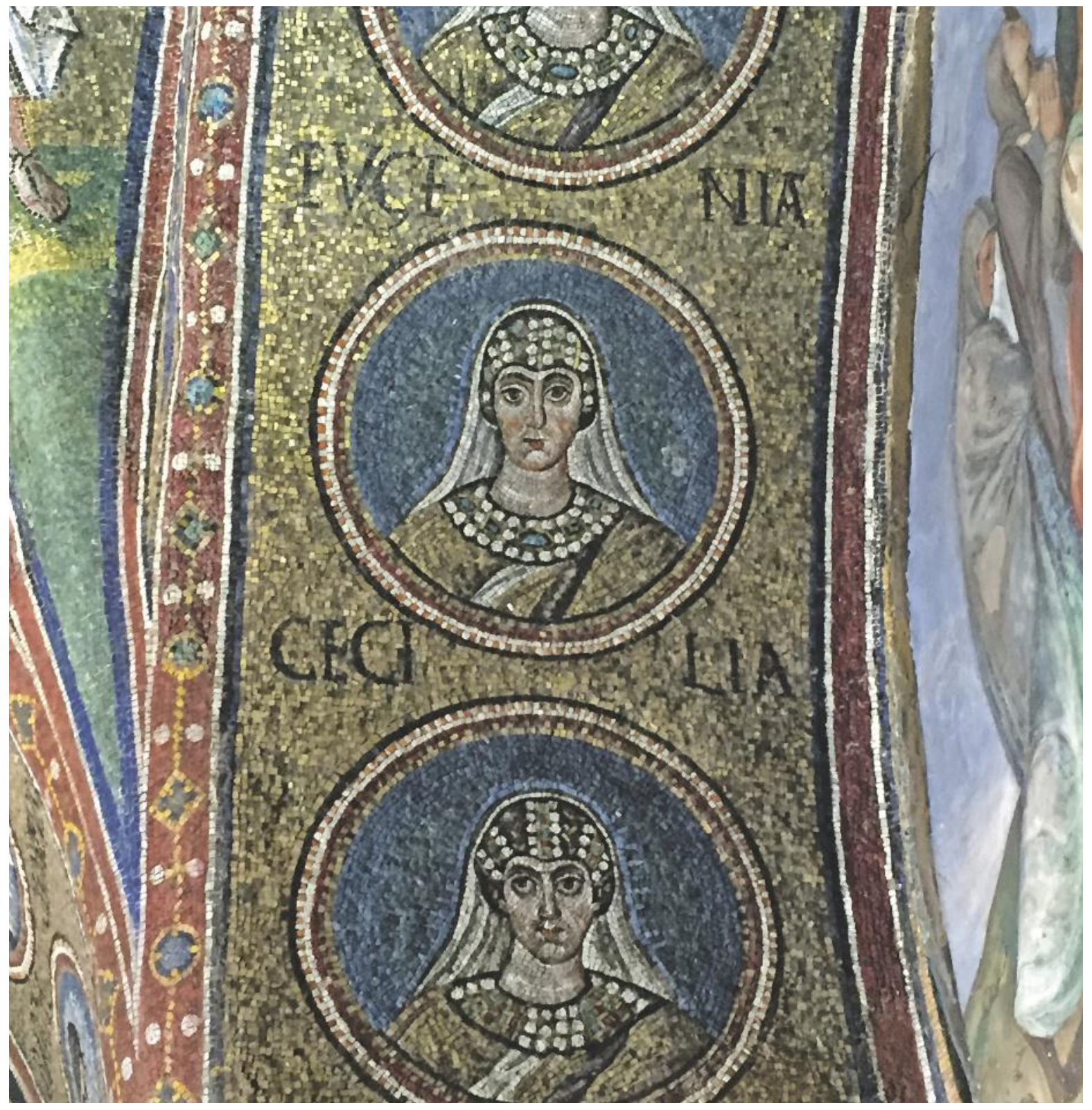

fig. 2

Ravenne, chapelle

du palais archiépiscopal,

mosaïque v. 495.

Photo Chloé Maillet. 
comme à Ravenne (fig. 2), adoptent des codes féminins non équivoques. La Vie a été féminisée et Eugène-Eugénie transformée en une sainte femme comme une autre, selon une iconographie codifiée comme sur les mosaïques ravennates. On n'a pas conservé avant Vézelay d'image de son procès, de ce moment précis de transition entre masculin et féminin.

D'où la réputation singulière du chapiteau, qui a suscité les commentaires des spécialistes des études de genre. Certaines études focalisées uniquement sur la vue frontale de l'image du chapiteau ont fondé leur analyse - fatalement partielle - sur une ombre au milieu du vêtement, interprétée comme une vulve féminine. Saisha Grayson associe sainteté féminine et ostension du corps, par référence aux images de femmes martyres souvent plus dénudées que les hommes (Grayson 2009). Surtout, ne sachant bien comment tirer parti de cette image inédite, Grayson lie l'ostension des attributs sexuels à des images apotropaïques. En effet, les images présentant des ostensions de vulves étaient fréquentes et couramment considérées comme des images apotropaïques, par exemple celle que l'on trouve sur la porta Tosa milanaise à la même époque (fig. 4).

Pourtant, ces images-objets sont très différentes de celle de Vézelay, autant par leur contexte, leur emplacement, que par ce qu'elles représentent. Le bas-relief de la porta Tosa a été sculpté sur la porte de la ville au moment où les Milanais luttaient pour l'indépendance communale. II s'agissait de repousser l'ennemi par ce geste de lever de jupe, aujourd'hui surprenant, que les historiens d'art et les anthropologues ont pourtant souvent remarqué ${ }^{\mathbf{1 1}}$. Les exemples de ce type d'images dans les églises sont postérieurs, notamment celle du mur nord de l'église Sainte-Radegonde à Poitiers (fig. 3), datant du XIII ${ }^{\mathrm{e}}$ siècle. Ce bas-relief prouve qu'il n'était pas impossible de montrer un corps explicitement dénudé au sein d'une église, du moment qu'il s'inscrivait dans un contexte spécifique. À Sainte-Radegonde, le personnage est en effet représenté à l'extérieur de l'église. L'image fait ainsi la transition entre l'intérieur et l'extérieur de l'édifice, sa fonction apotropaïque ne faisant alors aucun doute. Enfin, dans les deux cas évoqués ici, les femmes sont figurées manipulant leurs organes sexuels, avec un objet sur la porta Tosa, en écartant ses lèvres de ses doigts à Sainte-Radegonde, bien autrement donc, qu'Eugène-Eugénie à Vézelay.

Là en effet, l'image est située dans la nef, et Eugène-Eugénie se déshabille, mais il-elle montre ses seins, non sa vulve. Ce n'est que sur des images plus tardives, du XIV siècle, que le corps est entièrement dénudé. Un manuscrit du Speculum Historiale de Vincent de Beauvais, un autre légendier dominicain, présente trois images consacrées à Eugène-Eugénie, celle de son éducation à la philosophie, celle de son baptême en compagnie de Prothe et Hyacinthe et celle de son procès où on la voit écarter les pans de son vêtement pour dévoiler son corps nu (fig. 5). Les images de corps dénudés servaient parfois de preuves lors de procès jugeant des accusations liées à la sexualité (impuissance, «hermaphrodisme») 12. C'est donc dans ce cadre qu'intervient le déshabillage d'Eugène-Eugénie, en tant que preuve ultime apportée à la cour. Se déshabiller c'est faire la preuve par l'image et par la monstration, la preuve irréfutable. En cela, cette image d'Eugène-Eugénie, reproduite à plusieurs reprises au XIV siècle, se rapproche de celle, antique,
11. Sur ce sujet, voir Gil Bartholeyns, Pierre-Olivier Dittmar et Vincent Jolivet, Image et transgression au Moyen Âge. Paris, PUF, 2008.

12. Dans certains manuscrits du décret de Gratien (écrit en 1140), cause 33 , les miniatures montrent les organes sexuels dénudés en cas d'accusation d'impuissance du mari entraînant la rupture du lien conjugal. 


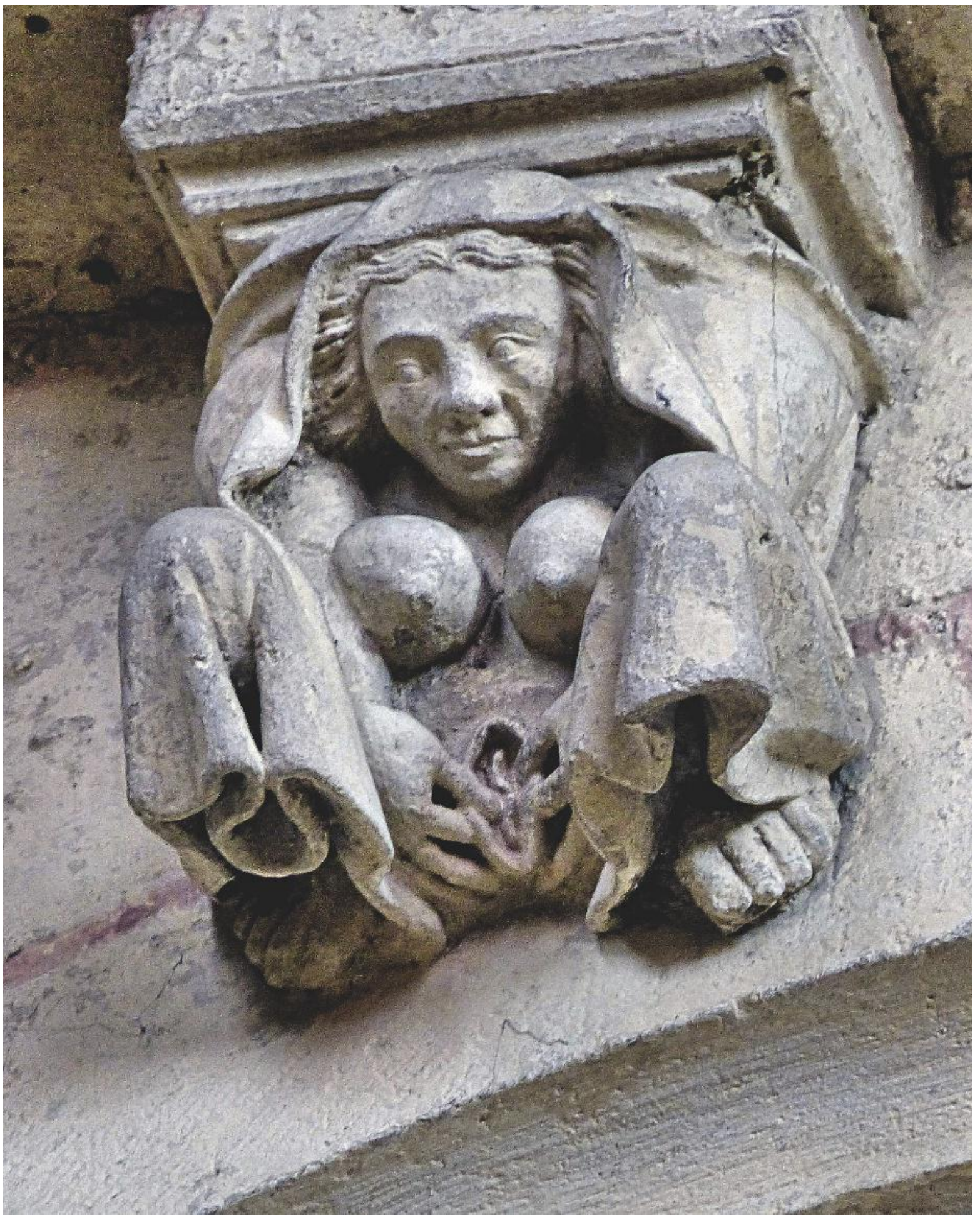




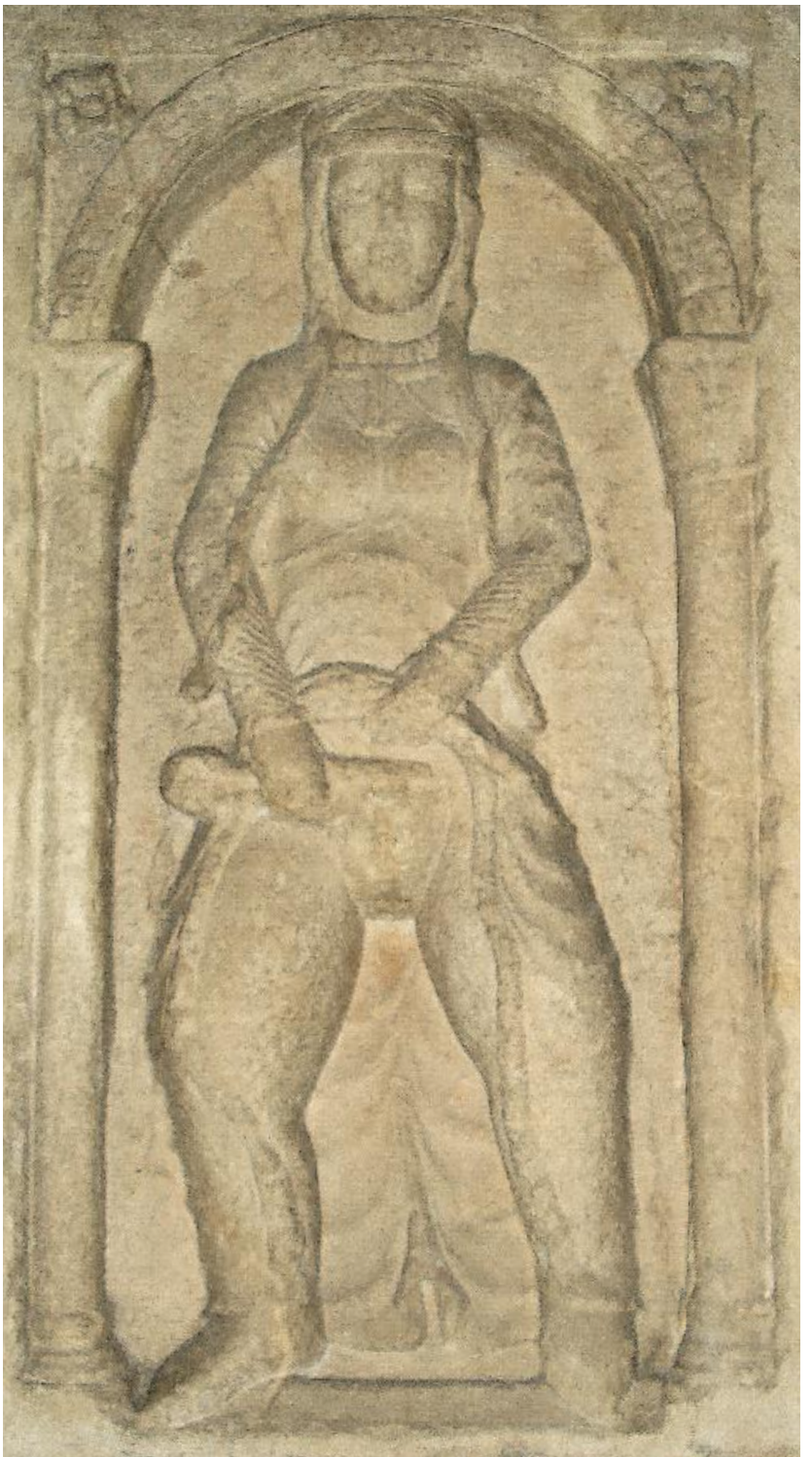

de Phryné, la courtisane aimée de Praxytèle, dont le corps était si beau qu'il est dit avoir servi de modèle à l'Aphrodite de Cnide. L'histoire rapportée par le pseudo-Plutarque voudrait qu'à son procès, son avocat, à court d'arguments, l'eût dévêtue et que la cour impressionnée par sa beauté, y vit une image de culte et l'absolut de ses crimes d'impiété ${ }^{13}$. Phryné devint ainsi une image de la preuve et du dévoilement de la vérité. Mais la comparaison ne fonctionne pas car dans l'image d'Eugène-Eugénie, ce sont les seins qui sont spécifiquement montrés pour preuve, et non la beauté d'un corps. Le corps d'Eugène-Eugénie n'apparaît pas comme doté de caractéristiques physiques attirantes, on y voit les os sous la poitrine. C'est celui d'un ascète, dévoilant une partie de son anatomie, le corps redoublé par son vêtement, puisqu'une suivante tend une robe féminine à la jeune personne qui a quitté

\section{page de gauche}

fig. 3

Détail du chapiteau.

Mur nord de la nef,

église Sainte-Radegonde,

Poitiers, XIII' siècle.

Droits réservés.

\section{page de droite}

fig. 4

La donna impudica,

Castello Sforzesco, porta

Tosa, Milan, XII siècle.

Photo Giovanni Dall'Orto, droits réservés.
13. Accusée de favoriser des cultes étrangers, Phryné fut acquittée. On peut se reporter au numéro des Cahiers des mondes anciens de 2016 sur l'image de Phryné comme modèle de vérité (Lehoux et Siron 2016). 


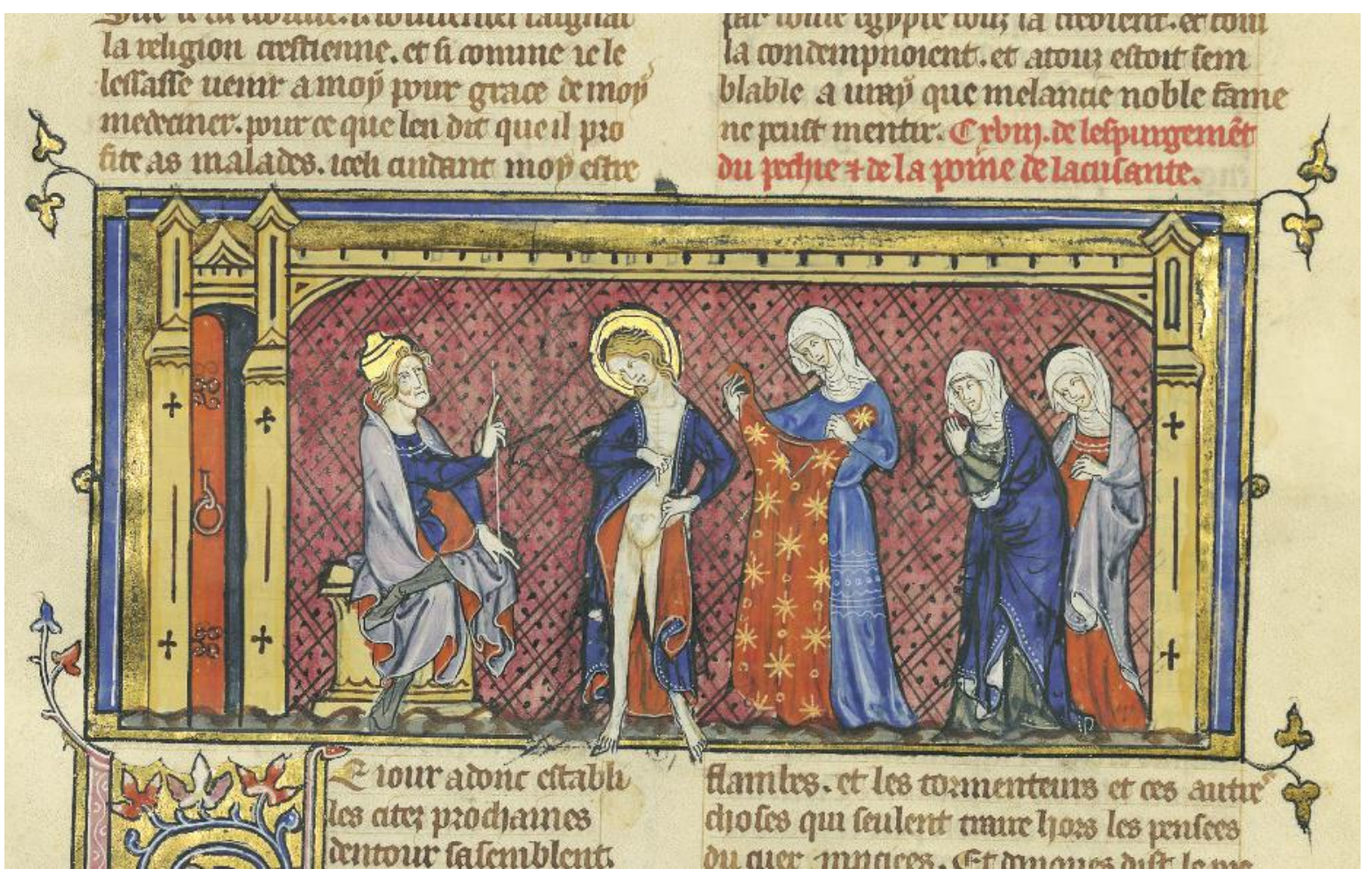

14. On en trouve une image presque identique dans un autre manuscrit du Speculum Historiale datant de 1370-1380 et conservé sous la cote Nouvelle acquisition française 15941 de la BNF.

15. Les détails sur l'église cités ci-après proviennent de ces trois thèses ainsi que des analyses archéologiques de Francis Salet (Salet 1995)

16. En 1117, le célèbre philosophe Pierre Abélard fut émasculé de force par l'oncle de son amie Héloïse pour avoir perverti la jeune fille (ils avaient eu un enfant, Astralabe, et s'étaient mariés en secret). La punition était sévère, administrée par vengeance familiale. Dans une lettre, Abélard raconte l'histoire de ses malheurs et fait finalement de ce châtiment une bonne chose, qui lui permit de ne plus être soumis à la tentation de la chair. II cite Origène (mort en 253), philosophe condamné pour s'être auto-émasculé, et soutient que lui-même n'étant pas l'auteur de l'acte, sa condition d'eunuque lui a permis, presque sans douleur, de s'abstraire de la chair. Si dans la perspective d'Origène, on peut rapprocher l'émasculation d'un idéal d'androgynie propre à l'Antiquité tardive dont Eugène-Eugénie serait un autre témoignage, chez Abélard, seul le péché de chair semble en cause (Abélard 1967).
I'habit d'homme ${ }^{\mathbf{1 4}}$. Sur le chapiteau de Vézelay qui précède de deux siècles le Speculum Historiale, il n'y a pas de corps dénudé mais seulement des «mamelles» (ubera ou mamilia en latin). Or cet organe caractérisant surtout l'allaitement n'était pas considéré comme érotique avant au moins le tournant entre le $\mathrm{XIII}^{\mathrm{e}}$ et le XIV ${ }^{\mathrm{e}}$ siècle, moment où l'on commence à évoquer le décolleté, qui fait son apparition dans les vêtements civils (Wirth 2013). C'est aussi probablement pour cette raison qu'on ne dévoile plus les seins d'Eugénie, potentiellement érotiques, alors qu'ils ne l'étaient pas au $\mathrm{XII}^{\mathrm{e}}$ siècle.

\section{Autour de lui-elle}

Pour regarder les seins d'Eugène-Eugénie, il faut être au cœur du dispositif ecclésial de Sainte-Marie-Madeleine de Vézelay. Cette église est complexe, énigmatique, et aucune archive ne nous est parvenue pour pouvoir éclaircir le contexte des choix iconographiques. Pas moins de trois thèses ont été consacrées à ses chapiteaux depuis les années 1990, qui ne coïncident pas dans leurs interprétations des images (Angheben 2003; Ambrose 2006; Huys-Clavel 2009) ${ }^{15}$. L'abbé en exercice à l'époque de la construction de la nef était Pierre le Vénérable, un des penseurs les plus influents de son époque - devenu peu après abbé de la toute-puissante abbaye de Cluny. Lui-même était très proche de Pierre Abélard qu'il accueillit après le scandale retentissant lié à son émasculation et son éviction du clergé ${ }^{16}$, et la controverse théologique qui l'opposa à Bernard, abbé de Clairvaux (1121). Des travaux y furent financés suite à la reconnaissance 
des reliques de la Madeleine vers 1040, qui a alors attiré des foules de pèlerins. Plus tard, en 1146, c'est là que Bernard fut envoyé par le pape pour prêcher la deuxième croisade. La foule attendue était si importante qu'il ne prêcha pas dans l'église - dont les dimensions étaient pourtant impressionnantes à l'époque, puisque l'église était la troisième en taille en France après Cluny et Saint-Sernin de Toulouse - mais sur la colline en descendant de la basilique, où fut édifiée une chapelle commémorative.

C'était donc bien au $\mathrm{XI}^{\mathrm{e}}$ siècle l'une des églises que l'on regarda de près. On l'oublia par la suite, après la découverte de reliques plus probables de Marie-Madeleine en Provence. Toutes les archives datant de sa construction furent détruites au $\mathrm{Xvl}^{\mathrm{e}}$ siècle. On ne sait donc plus très bien ni comment ni par qui elle fut construite, ce mystère venant alimenter les interprétations les plus variées.

\begin{tabular}{|c|c|}
\hline V. 1040 & Reliques de Madeleine à Vézelay. \\
\hline 1096 & Début des travaux de l'église romane. \\
\hline 1106 & $\begin{array}{l}\text { Fin des premiers travaux, le chœur et le transept sont } \\
\text { construits. Ils furent ensuite détruits par un incendie. }\end{array}$ \\
\hline 1120 & Début des travaux par Renaud de Semur. \\
\hline 1132 & Innocent II consacre l'église. \\
\hline V. 1140 & Construction du narthex. \\
\hline V. 1160 & Construction du chœur gothique. \\
\hline 1295 & $\begin{array}{l}\text { Authentification par Boniface VIII des reliques } \\
\text { de la Marie-Madeleine de Saint-Maximin-la-Sainte-Baume, } \\
\text { en Provence. }\end{array}$ \\
\hline
\end{tabular}

Eugène-Eugénie est sculptée sur un chapiteau qui constituait un point majeur du décor ecclésial à l'époque romane ${ }^{17}$. On a trouvé des restes de polychromie sur certains chapiteaux de Vézelay, et il n'est pas impossible que, comme pour beaucoup d'églises de l'époque, la sculpture des chapiteaux ait été complétée par un important décor peint aujourd'hui entièrement perdu.

Le chapiteau est aussi, pour reprendre la terminologie de Jérôme Baschet (2008), une image-objet, en trois, voire quatre, dimensions, puisqu'on ne peut voir ses trois faces que dans le temps, par le cheminement au sein de l'église (iter). Enfin, cette image a un locus précis, un lieu, au sein de l'espace ecclésial. En effet, un chapiteau à l'entrée du portail ne pourra avoir la même signification qu'un autre situé avant la croisée du transept et l'entrée dans le chœur, le lieu le plus sacré. La question de l'espace ecclésial est particulièrement complexe dans le cas de Vézelay car la nef étant la seule partie conservée de l'église romane d'origine, on ne sait par où on y entrait. Une fois l'avantnef (ou narthex) construite - immense à Vézelay, de trois travées -, les pèlerins passaient vraisemblablement par là pour pénétrer dans la nef, mais au début du $x^{\mathrm{e}}{ }^{\mathrm{s}}$ siècle, on ne peut le dire. De plus, on n'a gardé aucune trace des pratiques liturgiques de l'église; il est alors impossible de savoir si, comme dans beaucoup d'églises de l'époque, un chancel séparait l'espace réservé aux clercs de celui qui accueillait les laïcs et donc si le chapiteau d'EugèneEugénie était non loin de cette frontière, à un point de jonction de la circulation dans l'église.
17. Pour comprendre les nouvelles approches de l'art roman, fondées sur l'emplacement des chapiteaux, la déambulation, la liturgie et l'histoire sociale, on peut se référer aux travaux récents de Jérôme Baschet (Baschet 2008) ainsi qu'à l'ouvrage collectif écrit avec Jeanne-Claude Bonne et Pierre-Olivier Dittmar (Baschet, Bonne et Dittmar 2012b) ; et à l'ouvrage collectif dirigé par Cécile Voyer et Éric Sparhubert (2011).

\author{
ci-contre \\ fig. 5 \\ Vincent de Beauvais, \\ enlumineur Mahiet, \\ Le procès d'Eugène- \\ Eugénie, Speculum \\ Historiale v. 1335. \\ Paris, Bibliothèque \\ nationale de France, \\ ms 5080, fol. 154.
}




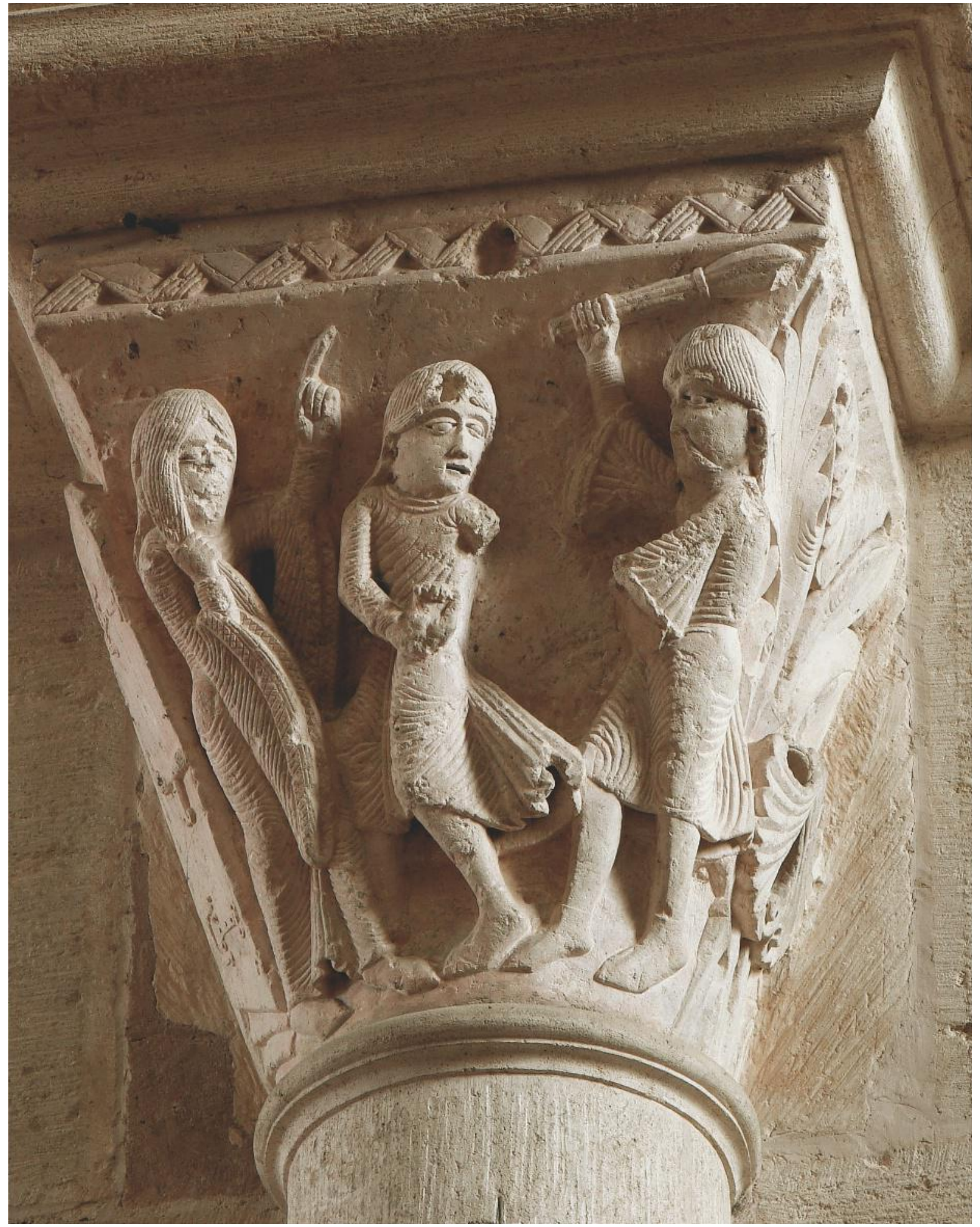




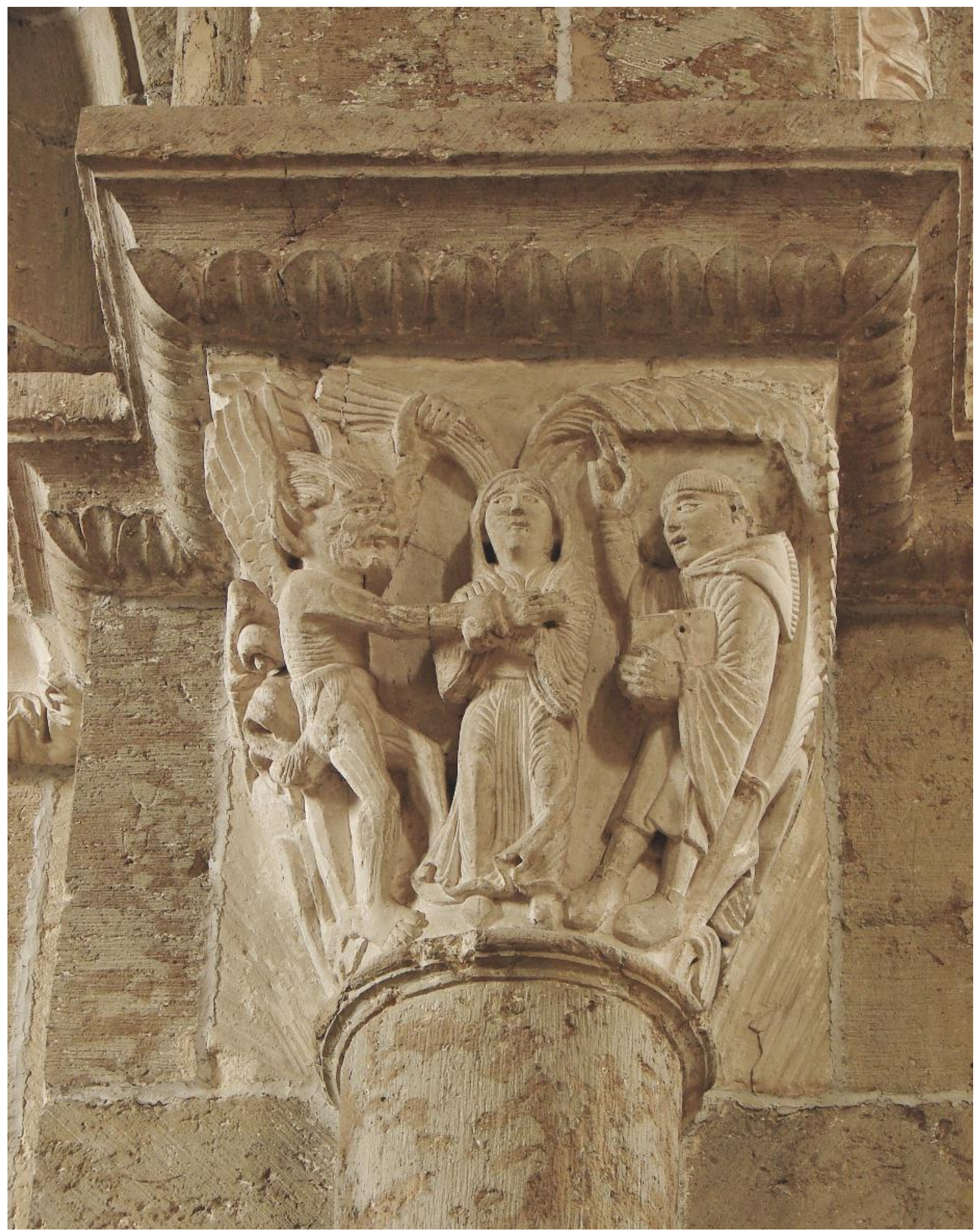


18. Selon Viviane Huys-Clavel (2009), au solstice d'été, on voit la lumière des baies dessiner des taches de lumières juste au centre de la nef. Lorsqu'on visite l'église, sa luminosité est frappante, comparée à nombre d'églises de l'époque.

19. Le chapiteau était autrefois interprété comme une vision de saint Antoine. Kirk Ambrose a proposé de l'associer à la chute de Simon le Magicien.

\section{page précedente de gauche}

fig. 6

Joseph accusé par la femme de Puthifar. Vézelay, basilique Sainte-MarieMadeleine, chapiteau 85, nord (C) CESCM Poitiers, Photothèque - S. Biay.

\section{de droite}

fig. 7

Tentation de saint Benoît. Vézelay, basilique

Sainte-Marie-Madeleine,

chapiteau 31, sud

(c) CESCM Poitiers,

Photothèque - S. Biay.
En circulant dans l'église, le chapiteau apparaît comme singulier: il s'agit de la seule "femme" sainte figurée dans toute la nef. Les autres personnages féminins représentés dans la nef sont des tentatrices, la femme de Putiphar de l'Ancien Testament qui tente Joseph, ou encore la tentation de saint Benoît (fig. 6 et 7). L'église est dédiée à Marie-Madeleine, la pécheresse repentie, mais aucune image de cette sainte n'a été sculptée dans le premier chantier de l'église (vers 1120-1138) - dont il ne reste aujourd'hui que la nef.

Le chapiteau est donc le seul qui montre une «femme" en tant que personnage positif. Remarquons que le chapiteau d'Eugénie se situe au nord, un emplacement important puisqu'il correspond généralement à l'entrée des églises (on passe d'abord par le nord avant d'aller vers le sud). Et même s'il est peu probable qu'il y ait eu un véritable portail au nord, il reste une petite porte qui pouvait au moins être utilisée par les moines. Or, en face de cette porte, on trouve justement le chapiteau d'Eugénie.

Si l'on observe ce chapiteau depuis cet emplacement, deux phénomènes sautent aux yeux. Premièrement, on remarque les effets de lumières propres à l'église dont on a longuement commenté l'ensoleillement particulier ${ }^{18}$. Viviane Huys-Clavel (2009) remarquait que le chapiteau d'Eugénie entrait en dialogue avec celui qui lui fait face - où apparaissent un basilic et un autre monstre, à connotation très négative - autour de la thématique de la dissimulation et du combat contre les forces du mal. Deuxièmement cependant, une observation sous une lumière normale révèle que les chapiteaux qui font face à celui d'Eugène-Eugénie sont dans l'ombre. En revanche, on voit aisément que ce chapiteau et ceux qui se suivent dans la nef, également éclairés, se répondent, formant une nouvelle série. Le chapiteau est ainsi mis en parallèle avec la pile suivante. On y trouve la chute de Simon le Magicien (chapiteau 62) ${ }^{19}$ et la tentation de saint Antoine (chapiteau 63) [fig. 8].

Cohérent d'un point de vue iconographique, ce rapprochement lie le chapiteau à la série d'images des pères du désert qui comprend aussi les funérailles de Paul Ermite (58, sur la même pile qu'Eugénie, à sa droite), un chapiteau non identifié qui pourrait être celui de la décapitation de Paul (60), des oiseaux becquetant du raisin (61) et la tentation de saint Antoine (63). Rapporter la vie d'Eugène-Eugénie à celle des pères du désert et à ce thème érémitique ferait sens, surtout si l'on est dans la partie de l'église correspondant davantage aux moines, qui à cette époque d'assimilation du grand projet de Réforme de l'Église (XI-XI" siècle) associaient culte de repentance (avec le développement du culte à la Madeleine repentie) et modèle érémitique (avec les pères du désert). II reste toutefois qu'elle serait la seule «femme» père du désert.

La scène se présente de manière très frontale contrairement aux autres chapiteaux de Vézelay dont très peu adoptent cette solution. Les chapiteaux étant faits pour être observés en marchant et en se déplaçant, la plupart en jouent, avec leurs éléments principaux placés aux angles, pour empêcher toute vision globale. Ce n'est pas le cas d'Eugène-Eugénie: le personnage principal est présenté frontalement, au milieu de la scène. Eugène-Eugénie est surmontée d'une palme (le martyre). Le végétal part du corps d'EugèneEugénie et les feuilles se replient en volutes sur les côtés du chapiteau. 
Elle est comme toute entière prise dans cette végétation luxuriante, qui la place dans la continuité des chapiteaux végétaux visibles juste après la série des pères (64, au musée lapidaire). Cette végétalité étudiée de manière novatrice par Jean-Claude Bonne 20 ne saurait être négligée:

Le végétal, en effet, «rend visible d'une certaine manière aux spectateurs l'aspect du paradis (paradysi Dei speciem floribus variis... quodammodo aspicientibus ostendendi [t]) " (Theophilus, De diversis artibus). Ce faisant, dans l'esprit des clercs au moins, la végétalisation de l'église est censée, sous certaines conditions liées à la nature et à la fonction spécifiques de ce locus orationis, transformer réellement, et non pas seulement d'une manière métaphorique, «la maison matérielle de Dieu » en une manière d'Eden provisoire qui est la promesse et l'anticipation du paradis à venir où devrait se réaliser une nouvelle et parfaite conjonction de l'âme et du corps, du matériel et du spirituel. (Baschet, Bonne et Dittmar 2012b, paragr. 21)

L'art roman des chapiteaux baigne entièrement dans cette masse végétale, qui s'est émancipée du chapiteau corinthien faisant référence à la romanité pour évoluer dans une foule de formes de feuillages différents. II est le soubassement de l'église et de sa puissance. Eugène-Eugénie fait littéralement naître cette palme, son martyre. Et les rinceaux que l'on voit apparaître aux côtés d'Eugène-Eugénie émergent dans des colonnes, comme dans la légende de naissance du chapiteau corinthien, maintes fois commentée par l'art roman.

Le chapiteau d'Eugène-Eugénie contraste ainsi avec celui auquel il ressemble le plus par isomorphisme, à savoir la tentation de saint Benoît. II se produit pourtant ici un basculement: le saint est placé au centre du chapiteau mais c'est de lui que naît la palme, annonce du futur martyre.

Le moine Eugène est torturé par une femme qui l'accuse à tort. Dans un milieu monastique, cette accusation inique peut être pensée comme un appel à la résistance du moine contre ses tentations, et une perspective de récompense. Eugène-Eugénie s'est découvert·e pour se justifier, et l'accusation est tombée. Est donc ici montré l'écart entre la femme accusatrice, mauvaise, et Eugène-Eugénie femme-homme et seul personnage féminin positif, saint·e, accusé·e à tort. Au milieu de cette série d'hommes du désert, isolé·e et seul·e saint·e de l'église, il ne peut d'un point de vue hagiographique être considéré comme une femme.

Mais cet homme a des mamelles. Ce contraste est étonnant pour nos yeux contemporains, mais pas du tout incompatible avec les discours qui commençaient à se répandre à ce moment-là autour des cisterciens.

Soyez des mères par votre amour, et des pères dans vos corrections. Soyez doux; point de dureté. Ménagez les châtiments, et montrez vos mamelles. Que votre sein soit rempli de lait, non point gonflé d'orgueil. (Bernard de Clairvaux 1996: sermon 23)
20. Jean-Claude Bonne comme Jérôme Baschet ont souvent relevé que toutes les études sur les chapiteaux romans excluaient les chapiteaux végétaux, laissant ainsi de côté près de la moitié du décor sculpté (Bonne 1985; Baschet, Bonne et Dittmar 2012a). 
fig. 8

Plan de l'église de Vézelay, avec l'emplacement des

chapiteaux autour de celui

d'Eugène-Eugénie, en face

d'une petite porte au nord.

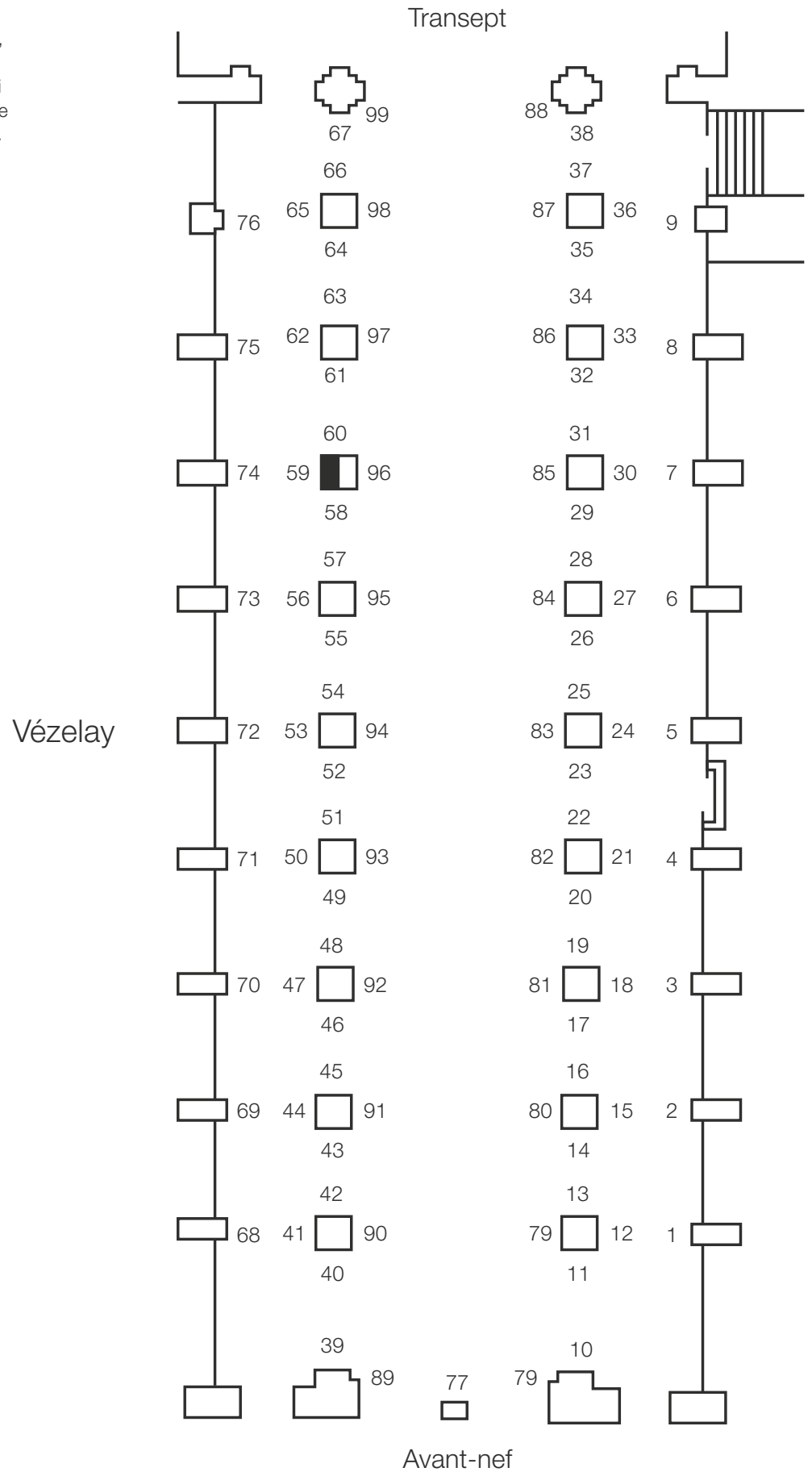

58: Funérailles de Paul Ermite 59: Procès d'Eugénie 60: Meurtrier de Saül?

61: Oiseaux becquetant du raisin 62: Chute de Simon le Magicien
63: Tentation de saint Antoine

73: Feuillages

74: Basilic

75: Repas de Paul et Antoine 
Discours qui prirent encore plus de poids avec les franciscains, eux-mêmes plus tard spectateurs de ce chapiteau car installés à Vézelay précocement - où se trouvait le premier couvent franciscain français:

Lorsqu'elle fut arrivée près de saint François, celui-ci sortit de sa poitrine une mamelle et lui dit: «viens, reçois et suce", et après qu'elle l'eut fait, le saint l'admonesta pour qu'elle suça une autre fois; et ce qu'elle suçait était si doux et délicieux qu'on ne pouvait l'expliquer en aucune façon ${ }^{21}$. (Vision de Claire d'Assise)

Cette image n'est donc pas incohérente dans un système où l'on verra aussi apparaître plus tard un saint Mamant, homme allaitant et protégeant la lactation féminine, mais encore inédite dans l'art roman. La conjonction entre virilité et caractéristiques féminines serait plutôt caractéristique d'une nouvelle forme de rapport avec le monde spirituel. Le personnage désigné comme sainte Eugénie était particulièrement valorisé au haut Moyen Âge comme modèle de sainteté féminine et virile, parfois associée à Radegonde ${ }^{22}$. II est possible qu'une image précédente ait existé notamment à l'abbaye de Varzy - dans la Nièvre, à 38 kilomètres, où était conservé un bras-reliquaire de «sainte Eugénie». Ceci expliquerait que cette Vie fût connue à Vézelay où une perméabilité avec les thèmes cisterciens est tangible, liée au fait que Pierre le Vénérable y était écolâtre au moment de la controverse entre clunisiens et cisterciens ${ }^{\mathbf{2 3}}$. Le discours cistercien tendrait plutôt à féminiser le masculin, selon la formule «Jesus as mother », popularisée par les études de Caroline Walker Bynum ${ }^{24}$. Le croisement entre tradition de virilité féminine et l'idée d'une féminisation des moines a pu permettre l'émergence de cette figure transgenre.

Abélard, proche de Pierre le Vénérable, et reçu à Vézelay à l'époque de ses déboires avec le clergé, mentionnait Eugène-Eugénie comme modèle de chasteté pour Héloïse. Selon l'analyse d'Abélard, le choix de changement de genre et d'habit était justifié par l'autorité abbatiale, car c'est Helenus qui aurait ordonné à Eugène-Eugénie de prendre l'habit monastique:

Bien plus, si des vierges fiancées, avant de s'unir charnellement à leurs maris, prennent la résolution d'embrasser la vie monastique et de renoncer à leur époux terrestre pour prendre le céleste époux, liberté leur en est laissée: ce qui n'a jamais été, que nous sachions, accordé aux hommes.

Quelques-unes furent même enflammées d'un tel zèle de chasteté, que non contentes de prendre, malgré la défense de la loi, un habit d'homme, elles se retirèrent parmi les moines, où l'éminence de leurs vertus les a rendues dignes de devenir abbé. Telle la bienheureuse Eugénie, avec la complicité de l'évêque Hélénus, que dis-je? sur son ordre, revêtit l'habit d'homme, et après avoir été baptisée par lui, fut admise dans un collège de moines. (Abélard et Héloïse 2000 : lettre 7)

Il est certain que cette image de saint-sainte moine aux mamelles est devenue un des thèmes les plus frappants de la vie d'Eugénie-Eugène que l'on retrouvera au XIII ${ }^{\mathrm{e}}$ siècle dans un panneau catalan venu d'une église de sainte Eugénie à Saga (fig. 9).
21. Fonti Francescane. Scritti et biografie di S. Francesco d'Assisi. Cronache et altre testimonianze del primo secolo francescano, Padoue, EMP : 2333 et sq., cité par Roberto Lionetti (Lionetti 1984).

22. Venance Fortunat $\left(\mathrm{V} \mathrm{I}^{\mathrm{e}} \mathrm{S}\right.$.) associait Eugénie à Radegonde sont il écrivit le panégyrique: voir Venance Fortunat, Opera poetica, Carminum liber VIII, carmen III, cité par Jane TibettsSchulenburg (TibbettsSchulenburg 2001: 161). Je remercie Cécile Voyer d'avoir attiré mon attention sur ce passage.

23. Les moines de Vézelay étaient favorables à la réforme du clergé, et la nef fut construite vers 1120-32 au cœur de la controverse sur l'application de la réforme monastique qui vit s'opposer clunisiens et cisterciens, Voir à ce propos: Bredero 1985: 145-47.

24 Sur la résurgence du thème de la maternité dans la spiritualité cistercienne il est fondamentale de se reporter aux études de Caroline Walker Bynum (Bynum 1982). 


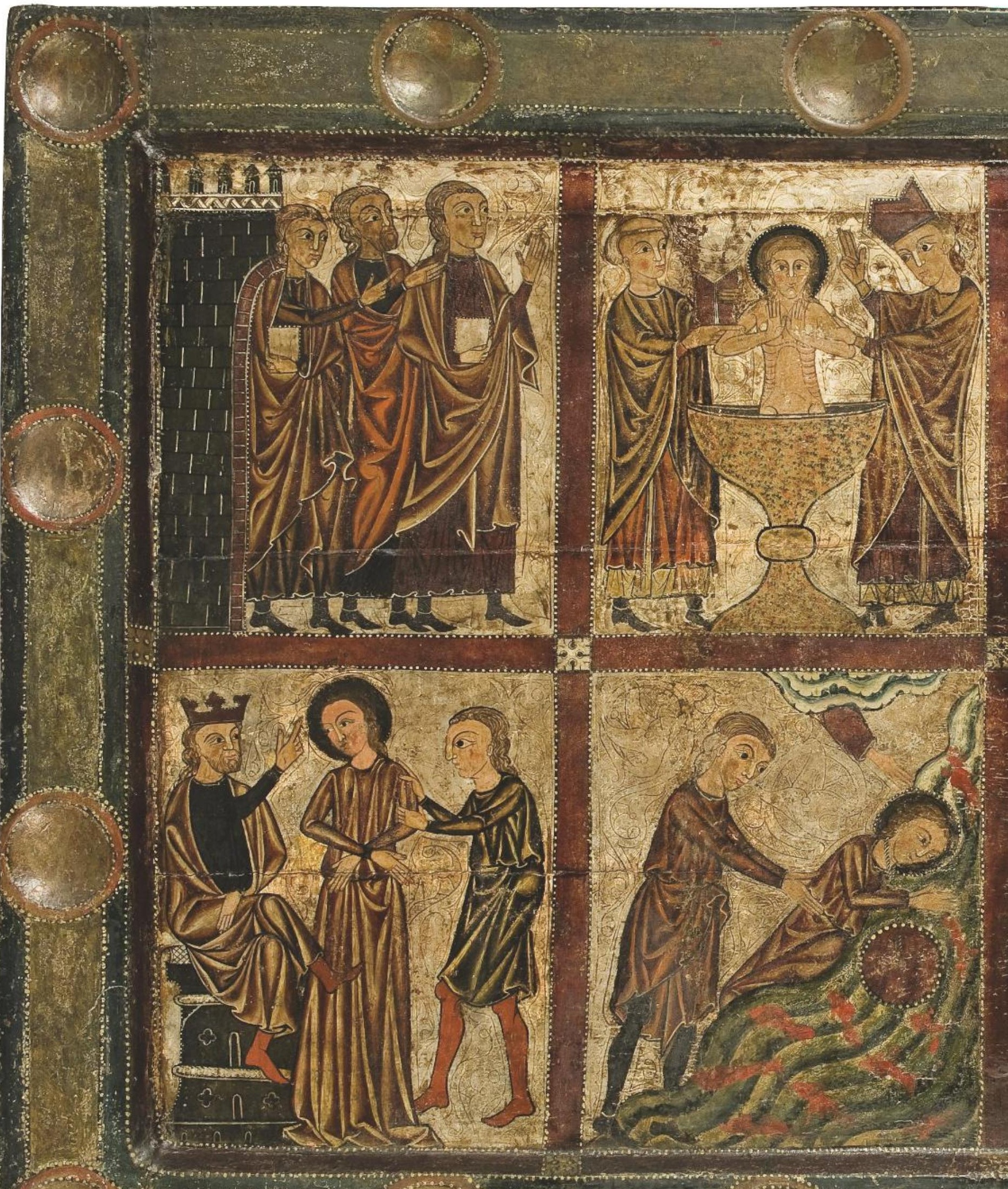


25. Selected Papers I. Romanesque Art. New York, George Braziller, 1977: 1-27.

26. Giovanni Paolo Maggioni (éd.), Legenda aurea. Florence, Sismel, 1998, chap. cxxx. On pourrait aussi traduire par "tu agis virilement" (Recte vir diceris quia cum sis femina viriliter agis). La vie originelle d'Eugénie proposait déjà la même formule: «Tu as raison de t'appeler Eugène car tu te conduis virilement, mais apprends que le Saint-Esprit ne m'a pas laissé ignorant que tu es Eugénie ", Vita Sanctae Eugeniae, Virginis ac martyris, A.A.S.S, décembre, 25 ; P.L., 21 , col. 1111.

\section{double page} précédente

\section{fig. 9}

Maître de Soriguerola, Frontal de sainte Eugénie. Panneau de la vie de sainte Eugénie provenant de Saga (Catalogne); en haut du panneau à droite, on retrouve la scène du procès d'Eugène-Eugénie (C) Photo Les Arts Décoratifs, Paris/Jean Tholance.
Le panneau en question joue de la multiplicité des scènes de la vie d'Eugène-Eugénie pour produire deux séquences narratives. Le registre supérieur montre Eugène homme, baptisé puis en habits monastiques. La scène du procès, en haut à droite dévoile sa poitrine et opère un basculement dans l'image de la sainte qui dès la scène suivante a tous les attributs féminins: cheveux longs et robe longue.

Le chapiteau roman, lui, utilise autrement ces motifs: le dévoilement de la poitrine sur un corps tout à fait masculin y donnant à voir une image ambivalente contrastée, particulièrement prisée dans l'art roman, comme l'avait bien remarqué Meyer Schapiro ${ }^{25}$. C'est ainsi que la question transgenre est entrée dans l'art roman majeur: comme union parfaite des opposés plutôt que comme transgression. C'est le début d'une nouvelle série iconographique qui explore en images les variabilités de ces transitions de genre.

Ce chapiteau de saintee Eugène-Eugénie, représentation singulière d'un.e saint·e moine à Vézelay, a dû être beaucoup regardé, beaucoup commenté. II faisait peut-être écho à l'époque à d'autres images aujourd'hui disparues. En tout cas, il atteste de manière visible que la problématique de la transition de genre était alors pensée et qu'Eugène-Eugénie est, comme il-elle le sera par la suite, associé.e aux Vies des pères du désert, un modèle érémitique en faveur à l'époque de la réforme monastique.

Ce chapiteau nous renseigne aussi sur la question du genre au sein de l'ensemble des images de l'église de Vézelay. Seule sainte de la nef, Eugénie tendrait à montrer que la seule "femme" positive dans l'ambiance clunisienne est considérée comme un homme. Et l'on sait les débats qui pouvaient avoir lieu à l'époque, alors qu'un clerc de grande renommée, Abélard, avait fait l'objet de scandale et fini eunuque. En 1140, Abélard condamné et excommunié fut accueilli par Pierre le Vénérable, l'ancien écolâtre de Vézelay, dont certains pensent qu'il aurait contribué à penser le décor sculpté. Abélard, théologien «star » de l'époque, avait commenté le parcours d'EugèneEugénie, qu'il défendait comme modèle auprès d'Héloïse dans ses lettres.

On pourrait croire qu'il ne s'agissait que de métaphores, comme celle de l'allaitement masculin défendu par Bernard de Clairvaux; des images fortes, mais purement théoriques. De fait, l'histoire d'Eugénie n'a que peu de sources historiques avérées, et la double circulation de sa vie à Rome et Alexandrie, en fait une hagiographie légendaire. Pourtant, dans les années 1180, est apparue à Schonäu dans l'Empire, un moine vénéré, dont on découvrit au moment de sa mort qu'il avait des seins et une vulve (Hotchkiss 1996: chap. III). Une histoire contemporaine de Vézelay, dont on peut très raisonnablement penser qu'elle est historique. Le texte racontant la vie de ce moine et rédigé le jour du décès de ce dernier par Engelhard parle d'une personne transgenre dont on a dû «transitionner» le genre post mortem - genus masculinum in femininum transivit.

Hélénus l'abbé très pieux d'Eugène-Eugénie qui l'accueillit en son monastère lui disait selon la vita latine reprise par La Légende dorée:

Tu as raison de te dire homme, car bien que tu sois une femme, tu te comportes comme un homme ${ }^{26}$. 
Il reste qu'à cette époque porter les habits de l'autre genre était puni par la loi et la transidentité, bien qu'elle puisse se référer à un idéal de vie remontant au paléochristianisme, ne pouvait être vécue que de manière exceptionnelle, illicite ou cachée. Cela ne veut pas dire qu'elle n'existait pas. Ces cas restent l'exception dans le monde du $x^{e}{ }^{e}$ siècle mais ils peuvent être envisagés comme un véritable laboratoire du genre. Le genre avait alors à faire avec des qualités qui pouvaient être attribuées à des personnes assignées femmes ou hommes (chasteté masculine et luxure féminine). Le monde médiéval n'était pas encore naturaliste, et enracinait sexe et genre dans un récit (la Création), plutôt que dans un certain rapport à la biologie $\mathbf{2 7}^{\mathbf{2 7}}$
27. Depuis les années 1990, des chercheuses issues de la biologie comme Anne FaustoSterling et Donna Haraway ont critiqué la binarité sexuelle que ni les organes, ni les chromosomes, ni les caractères sexuels secondaires ne parviennent à justifier, mais cette idée peine à s'imposer publiquement (Haraway 1991; Fausto-Sterling 2012 ; Fausto-Sterling et Molinier 2013).
ALHOMA-CRH (EHESS)

ESBA Angers (TALM) chloemaillet@yahoo.com

\section{Remerciements :}

Ce travail a bénéficié de la relecture attentive et des conseils de Laurence Hérault, Sylvie Steinberg, Georges Sideris, Cécile Voyer, Jessica De Largy Healy, Frédéric Keck, qu'ils en soient chaleureusement remerciés. Lee Rozada a beaucoup participé au difficile travail de relecture et d'adaptation du langage, et il sait combien je lui dois. Je reste entièrement responsable des interprétations et des éventuelles inexactitudes. 

2018 "Femmes moines, transgenres et gothiques. Points de vue médiévaux" [en ligne], Fabula LHT 20, disponible sur: www.fabula.org/ lht/20/maillet.html.

\section{Pastoureau, Michel}

2012 "Les cornes, les poils, les oreilles et la queue. Se déguiser en animal dans l'Occident médiéval ", in Estremità e escrescenze del corpo/ Extremities and Excrescences of the Body. Florence, Sismel: 3-24.

\section{Perret, Michèle}

1985 "Travesties et transsexuelles Yde, Silence, Grisandole

Blanchandine ", Romance notes 3 (25) : 328-340.

\section{Rolker, Christof}

2014 "The two laws and the three sexes: Ambiguous bodies in canon law and Roman law (12th to 16th centuries) ", Zeitschrift Der Savigny-Stiftung fûr

Rechtsgeschichte 131: 178-231.

\section{Salet, Francis}

1995 Cluny et Vézelay. L'CEuvre des sculpteurs. Paris, Société française d'archéologie.

\section{Scott, Joan W.}

2010 "Le genre: une catégorie

d'analyse toujours utile? ",

Diogène 225: 5-14.

\section{Sideris, Georges}

2010 "La trisexuation à Byzance ", in Michèle Riot-Sarcey (dir.), De la différence des sexes. Le Genre en histoire. Paris, Larousse.

\section{Sparhubert, Éric et Voyer, Cécile (dir.)}

2011 L'Image médiévale. Fonctions dans l'espace sacré et structuration de l'espace cultuel. Turnhout, Brepols.

\section{Steinberg, Sylvie}

2001 La Confusion des sexes.

Le Travestissement de la Renaissance à la Révolution. Paris, Fayard.

\section{Théry, Irène et Meroyer,} Anne-Marie

2014 Filiation, origines, parentalité. Le Droit face aux nouvelles valeurs de responsabilité générationnelle. Paris, Odile Jacob.

\section{Tibbetts Schulenburg,} Jane

2001 Forgetful of their Sex. Female Sanctity and Society, ca 500-1100. Chicago/Londres, The University of Chicago Press.

\section{Wirth, Jean}

2013 "Le sein féminin au Moyen Âge ", in L'image du corps au Moyen âge. Florence, Sismel Edizioni del Galluzo: 73-94.

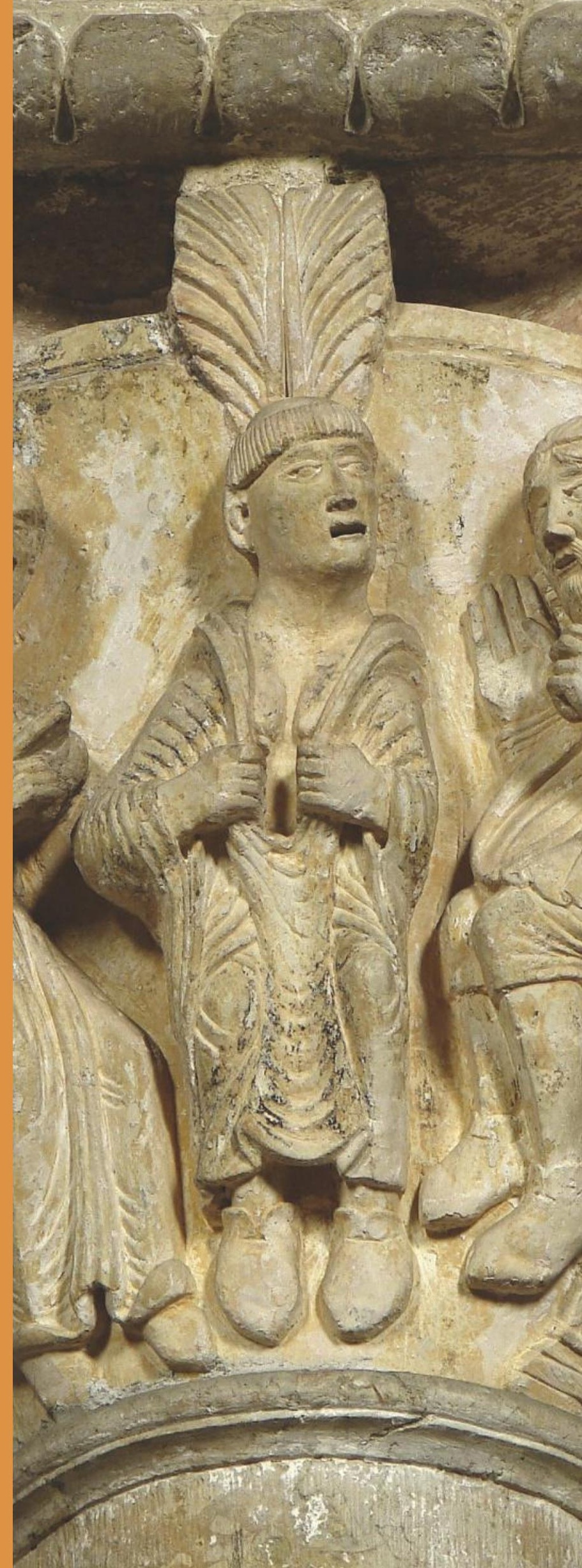

